\title{
Supraclavicular Scrofuloderma: A Diagnostic Challenge without Apparent Clinical Manifestations of Tuberculosis
}

\author{
Yusuke Yoshioka ${ }^{a} \quad$ Takeshi Namiki $^{a}$ Tsukasa Ugajin ${ }^{a} \quad$ Keiko Miura ${ }^{b}$ \\ Hiroo Yokozeki ${ }^{a}$ \\ aDepartment of Dermatology, Graduate School of Medical and Dental Sciences, Tokyo \\ Medical and Dental University, Tokyo, Japan; ${ }^{b}$ Department of Pathology, Graduate School of \\ Medical and Dental Sciences, Tokyo Medical and Dental University, Tokyo, Japan
}

\author{
Keywords \\ Supraclavicular $\cdot$ Scrofuloderma $\cdot$ Tuberculosis
}

\begin{abstract}
Scrofuloderma is one of the cutaneous manifestations of tuberculosis and usually occurs when underlying tuberculosis such as lymphadenitis directly involves the skin. However, the diagnosis of scrofuloderma without other apparent clinical manifestations of tuberculosis is sometimes challenging. A 27-year-old male from Bangladesh presented with a dome-shaped tumor on his right clavicle. MRI showed a high-density area that expanded from the lymph nodes between the internal jugular vein and the common carotid artery into the tumor on his right clavicle. Bacterial examinations of cultures from the tumor detected Mycobacterium tuberculosis. Further examinations for pulmonary tuberculosis including chest X-ray and computed tomography did not detect any lesions suggestive of pulmonary tuberculosis. From those examinations, the diagnosis of scrofuloderma was made. We emphasize the importance of recognizing a variety of clinical manifestations of tuberculosis including scrofuloderma due to the recent increase of immigrants from developing countries.
\end{abstract}

(C) 2021 The Author(s).

Published by S. Karger AG, Basel

\section{Introduction}

Scrofuloderma is one of the cutaneous manifestations of tuberculosis and usually occurs when underlying tuberculosis such as lymphadenitis directly involves the skin [1-3]. However, scrofuloderma without palpable lymphadenopathies and other clinical manifestations of tuberculosis are relatively rare, and its diagnosis is sometimes challenging. Here, we report a 

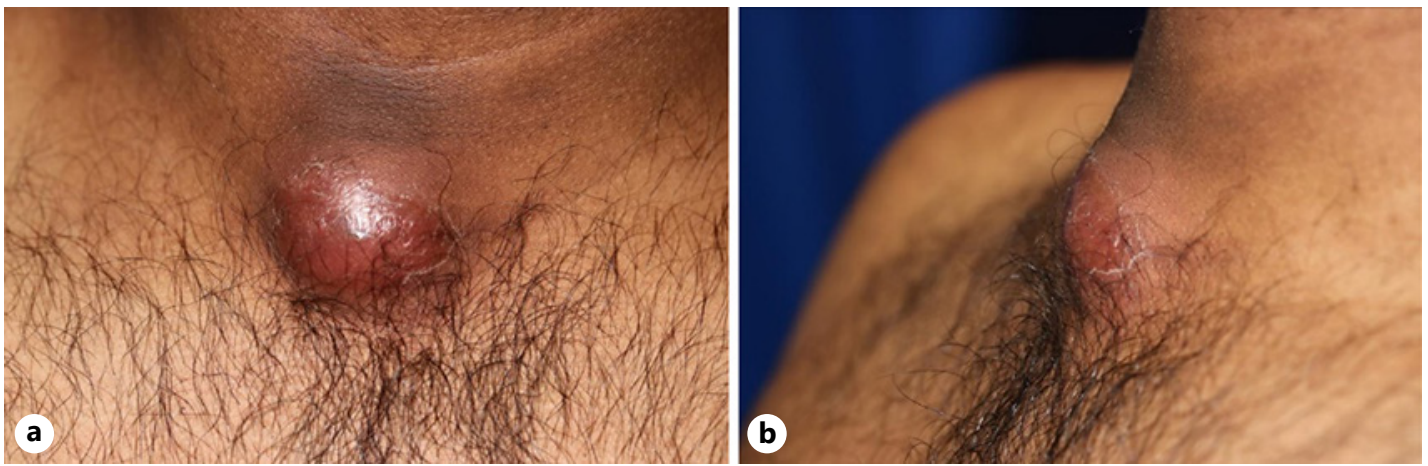

Fig. 1. a Frontal view of the tumor, a $41 \times 39 \mathrm{~mm}$ red dome-shaped tumor on the patient's right clavicle. Redness and swelling were found at the tumor. A slight fistula was also found in the center of the tumor. $\mathbf{b}$ Oblique view of the tumor; a slight scale was found on the tumor.

case of supraclavicular scrofuloderma without apparent clinical manifestations of tuberculosis. This case is intriguing and instructive in terms of the lack of an apparent infectious focus of tuberculosis including lymphadenopathies and pulmonary tuberculosis from clinical manifestations.

\section{Case Report}

A 27-year-old male from Bangladesh presented with an indurated subcutaneous tumor on his right clavicle. The patient had moved to Japan from Bangladesh 8 months earlier and had recognized a nodule on his right clavicle 2 months before. The nodule had gradually enlarged and sometimes produced a discharge. There was no family history of tuberculosis. Physical examination revealed a $41 \times 39 \mathrm{~mm}$ red dome-shaped tumor with slight scales on his right clavicle. A slight fistula was found in the center of the tumor (Fig. 1a, b). There was no apparent lymphadenopathy at his neck or axillae. Laboratory investigations revealed the following: leukocyte count: $8,000 / \mathrm{mm}^{3}$ (normal: $4,000-8,500 / \mathrm{mm}^{3}$ ), eosinophil count: 120 / $\mathrm{mm}^{3}$ (normal: $100-300 / \mathrm{mm}^{3}$ ), hemoglobin: $13.0 \mathrm{~g} / \mathrm{dL}$ (normal: $11.8-15 \mathrm{~g} / \mathrm{dL}$ ), platelets: $24.3 \times 10^{4} / \mu \mathrm{L}$ (normal: $13-30 \times 10^{4} / \mu \mathrm{L}$ ), C-reactive protein: $0.03 \mathrm{U} / \mathrm{mL}$ (normal: $<0.14 \mathrm{U} /$ $\mathrm{mL}$ ), and tuberculosis-specific interferon-gamma release assay: positive (normal: negative). MRI revealed a high-density area that expanded from the lymph nodes between the internal jugular vein and the common carotid artery into the tumor on his right clavicle (Fig. 2a). Histopathological examination revealed dense infiltrates of lymphocytes and histiocytoid cells intermingled with eosinophils in the dermis and subcutaneous tissues (Fig. 2b). Some of the foci of those dense infiltrates showed aggregates of histiocytoid cells with lymphocytes at the periphery (Fig. 2c). Some histiocytes fused to form epithelioid giant cells (Fig. 2d). There was no lymphoid structure. Bacterial examinations of cultures from the tumor detected $M y c o-$ bacterium tuberculosis. Tissue culture also detected Mycobacterium tuberculosis, but bacterial examinations from the sputum did not detect Mycobacterium tuberculosis. Further examinations for pulmonary tuberculosis including chest X-ray and computed tomography did not detect any lesions suggesting pulmonary tuberculosis. From those examinations, the diagnosis of scrofuloderma without pulmonary tuberculosis was made. Oral administration of rifampicin $450 \mathrm{mg} /$ day, isoniazid $300 \mathrm{mg} /$ day, ethambutol $750 \mathrm{mg} /$ day, and pyrazinamide $1,200 \mathrm{mg} /$ day reduced the tumor size, and the tumor had almost disappeared after 6 weeks of the initiation of that therapy.

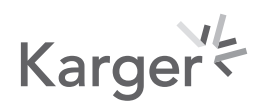



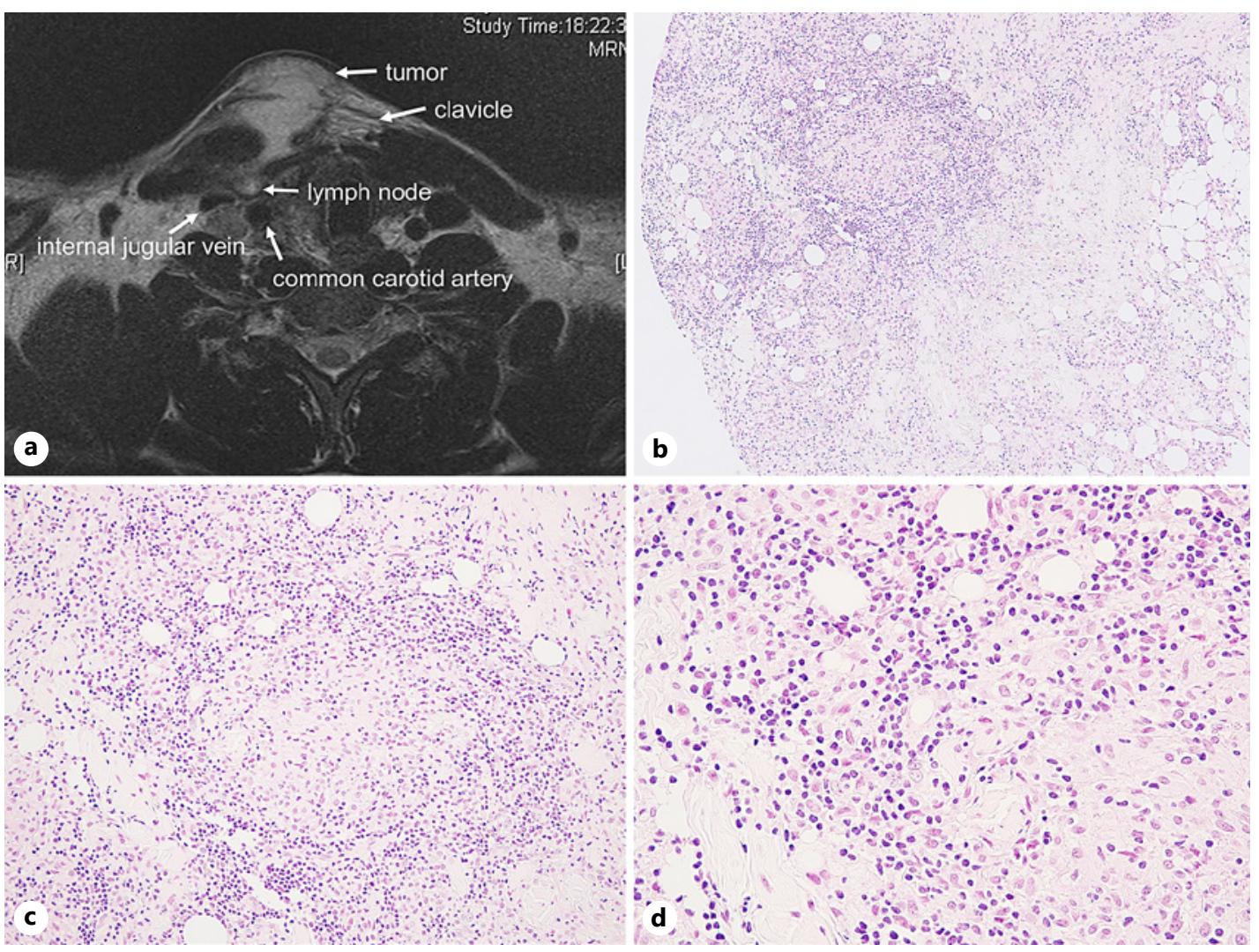

Fig. 2. a MRI showing a high-density area from the lymph nodes between the internal jugular vein and the common carotid artery into the tumor on the right clavicle. b Histopathology showing dense infiltrates of lymphocytes and histiocytoid cells intermingled with eosinophils in the dermis and subcutaneous tissues (H\&E stain; original magnification, $\times 100$ ). c Some of the foci with those dense infiltrates showed aggregates of histiocytoid cells with lymphocytes at the periphery (H\&E stain; original magnification, $\times 200$ ). d Some histiocytes fused to form epithelioid giant cells (H\&E stain; original magnification, $\times 400$ ).

\section{Discussion/Conclusion}

Scrofuloderma usually occurs at areas with underlying lymph nodes such as the neck. The underlying focus of tuberculosis in lymph nodes (lymphadenitis) sometimes extends into the overlying skin and is diagnosed as scrofuloderma [1]. This pathogenesis parallels the fact of the low incidence of scrofuloderma at the clavicle, where no apparent lymph nodes exist between the bone or joint and the skin [4]. In our case, the scrofuloderma was located above the right clavicle near the right sternoclavicular joint. We speculate from the MRI findings that this location may indicate scrofuloderma in our case extending from underlying lymph nodes in deep neck structures due to no apparent foci of tuberculosis in the patient's body other than this lesion of scrofuloderma on the clavicle. Our case is also important to caution dermatologists in developed countries to recognize a variety of scrofuloderma such as this rare case due to the recent increase in the number of immigrants from developing countries into developed countries. A recent survey of the incidence of tuberculosis worldwide revealed a huge difference of incidence between developing countries and developed countries [5]. This fact suggests that dermatologists in developed countries, who have less experience in seeing scrofuloderma, may have an opportunity to encounter a variety of scrofuloderma in patients from developing countries. The recognition of a 
variety of scrofuloderma has a critical importance for the accurate diagnosis of extrapulmonary tuberculosis.

\section{Statement of Ethics}

The authors have obtained written informed consent from the patient for the publication of this case report. This work was conducted in accordance with the Declaration of Helsinki.

\section{Conflict of Interest Statement}

The authors have no conflicts of interest to declare.

\section{Funding Sources}

The authors have no funding source to declare.

\section{Author Contributions}

Y.Y., T.N., and H.Y. drafted the manuscript. H.Y. and T.U. revised the manuscript critically. Y.Y., T.N. T.U., and K.M. were involved in the conception of this manuscript. K.M. performed the histological analysis and wrote the descriptions regarding histology. All the authors gave final approval of the latest version of this manuscript.

\section{References}

1 DeKlotz C, DeKlotz T. Images in clinical medicine. Scrofuloderma. N Engl J Med. 2012 Jun;366(23):2215.

2 Otto AI, Harsing J, Herjavecz I, Kiss M, Karpati S. Scrofuloderma associated with granuloma annulare-like lichen scrofulosorum. Acta Derm Venereol. 2009 Nov;89(6):640-2.

3 Müller H, Eisendle K, Zelger B, Zangerle R. Bilateral scrofuloderma of the axilla masquerading as hidradenitis suppurativa. Acta Derm Venereol. 2008;88(6):629-30.

4 Tan WP, Tang MBY, Tan HH. Scrofuloderma from the acromioclaviclular joint presenting as a chronic ulcer in an immunocompetent host. Singapore Med J. 2007 Sep;48(9):e243-5.

5 World Health Organization. Institutional repository for information sharing. Global tuberculosis report; 2018. https://apps.who.int/iris/handle/10665/274453. 